Asian-Australasian Journal of

Food Safety and Security

ISSN 2523-1073 (Print) 2523-2983(Online)

www.ebupress.com/journal/aajfss

\title{
Article
}

\section{Colistin residue in broiler: detection in different growth stages}

Naima Islam Bristy ${ }^{1}$, Sajal Das ${ }^{1}$, Zakaria al Noman ${ }^{1}$, Jannatul Ferdous ${ }^{1}$, Sabbya sachi $^{1}$, S. M. Lutful Kabir ${ }^{2}$ and Mahmudul Hasan Sikder $^{1} *$

${ }^{1}$ Department of Pharmacology, Bangladesh Agricultural University, Mymensingh, Bangladesh

${ }^{2}$ Department of Microbiology and Hygiene, Bangladesh Agricultural University, Mymensingh, Bangladesh

*Corresponding author: Professor Dr. Md. Mahmudul Hasan Sikder, Department of Pharmacology, Bangladesh Agricultural University, Mymensingh-2202, Bangladesh. Phone: $\quad+8801766677178$; E- mail: drmsikder@bau.edu.bd

Received: 25 April 2019/Accepted: 26 May 2019/ Published: 30 May 2019

\begin{abstract}
Indiscriminate use of antibiotics in broiler could lead to residue accumulation in food chain. We conducted a study to detect colistin residues in broiler meat in different growth stages, collected from different upazilas of Mymensingh division. A total of 108 samples, were collected from Fulbaria, Mymensingh Sadar, and Trishal Upazila of Mymensingh district and Nakla Upazila of Sherpur district. From each farm, nine samples were collected at the three different growth stages: 14-20 days (Early:E), 21-27 (Middle:M) days and 28-35 (Late:L) days, respectively. Thin layer chromatography (TLC) was used for the qualitative detection colistin residues in broiler meat. A questionnaire survey was carried out with the farmers from whom the samples were collected. Most of the farmers' had varying degree of knowledge about biosecurity, withdrawal period and residue of antibiotic, and academic qualification varies from primary to graduate level. Out of 108 samples, $50 \%$ (18 out of 36) samples were positive for TLC in early samples, whereas $66.67 \%$ (24 out of 36) samples for middle and 50\% (18 out of 36) for late collected samples. Upazila-wise, the highest concentration was detected in Mymensingh sadar and Nakla (66.67\%) and lowest in Fulbaria (33.33\%). These result indicates that colistin are being misused in poultry industry especially in middle days of broiler life and withdrawal periods are not maintained when broiler are marketed. Being a Reserve group antibiotics, measures are needed to ensure human safety regarding colistin residue in poultry industry.
\end{abstract}

Keywords: antibiotics residues; broiler meat; colistin; growing stage; thin-layer chromatography

\section{Introduction}

The poultry sub-sector is an important avenue in fostering agricultural growth and reduce malnutrition for the people in Bangladesh (Da and Rankin, 2014). Poultry meat contributes 37\% of the total meat production and about 22-27\% of total animal protein in the country (Prabakaran, 2003).

Poultry antibiotics are widely used not only to treat diseases but also to maintain health, promote growth and enhance feed efficiency (Okerman et al., 2007). Modern animal production practices in Bangladesh and in many countries are associated with regular use of antimicrobials, potentially increasing selection pressure on bacteria to become resistant.

However, antibiotics that are being used in poultry, could lead to deposit of drug residue in poultry products, when use indiscriminately that may contribute adverse health hazard to the consumers (Sarker et al., 2018). Moreover, lack of guidance, failure to notice drug withdrawal period, lack of consumer awareness could also contribute antibiotic residues in poultry edible tissue (Singh et al., 2014). The residue could further lead to the emergence of antibiotic resistance bacteria in the environment and living system (Nisha et al., 2008; Hasan et al., 2014).

Colistin is a polypeptide antibiotic that is used in veterinary medicine as the sulphate salt for oral preparations and as the methane sulphonate for parental administration (Weiss et al., 2007). It is classified as "Reserve 
group" antibiotics and no residue of colistin is accepted in poultry meat. There are scarcity of information regarding colistin residue in poultry meat in Mymensingh division (Sarker et al., 2018) and no study yet designed to access the residual presence in broiler meat at different growth stages to understand prescribing and usage pattern of colistin. The study was undertaken to determine the presence of colistin residue in raw broiler meat at different growth stages at Mymensingh division.

\section{Materials and Methods}

\subsection{Experimental design}

We conducted the experiment Firstly to assess farmers using questionnaire survey and secondly, screening of colistin residue in poultry meat by TLC. The survey was conducted by a semi-structured questionnaire for assessing (i) the farmers' educational level, (ii) Knowledge of residue, (iii) Storage of drugs and (iv) the purpose of antibiotic usage.

\subsection{Study area}

Samples were collected from Fulbaria, Mymensingh Sadar, and Trishal Upazila of Mymensingh district and Nakla Upazila of Sherpur district during the period of August - September, 2017. The pre-prepared questionnaire was filled up properly before sample collection. The questionnaire was designed to assess farmers' perspective regarding awareness and biosecurity program as well as educational status.

\subsection{Collection of sample}

A total of 108 broilers were collected from four farms. From every farm 27 broilers were collected: at the age of 14-20 days, 21-27 days and 28-35 days. Each sample was dressed and placed into a plastic zipper bag transferred to laboratory by ice bag plastic container and stored at $-18^{\circ} \mathrm{C}$ until extraction.

\subsection{Chemical and standard drugs}

For extraction, HPLC grade methanol (Merck-Germany), trichloracetic acid (TCA), diethyl ether and acetone (RCI Labscan-Thailand) was used. Colistin was obtained from Sigma-Aldrich via Renata Limited, Bangladesh. The standard for colistin were prepared by dissolving $0.1 \mathrm{gm}$ of powder in $4 \mathrm{~mL}$ solution of methanol as cited by (Hussain et al., 2005). Standard solution was stored in $-4^{\circ} \mathrm{C}$ and fresh solution was prepared for every month.

\subsection{Sample preparation for TLC}

The experiment was conducted as described by Popelka et al., 2005. Stored meat sample was cut into small pieces and grinded properly with a grinder. Then $4 \mathrm{mg}$ sample ware weighted with weight box and taken into a falcon tube which contains $10 \mathrm{ml}$ phosphate buffered saline and mixed by vortex. After proper mixing $2 \mathrm{ml} 30 \%$ trichloroacetic acid (TCA) was added and mixed properly by vortexing. The samples were then centrifuged at $6000 \mathrm{rpm}$ for 20 minutes with the help of automatically time regulated centrifuge machine. At least $2 \mathrm{ml}$ supernatant were taken. The supernatant was extracted with an equal volume of diethyl ether to perform defatation. The mixture was then kept for 8-10 min to become into a separate layer and $1 \mathrm{ml}$ was taken from the bottom of the falcon tube. Then placed into TLC plate and observed.

\subsection{Pointing, running and detection on TLC plate}

For performing TLC, TLC plate (MN-Germany), TLC tank and UV detection box (UV light: F18W-Germany) were used. We have performed TLC according to Tajick and Shohrel, (2006) with few modifications. Firstly, TLC plate was cut into a size of $4 \times 5 \mathrm{~cm}$ from $20 \times 20 \mathrm{~cm}$. A straight line was put across the plate approximately $2 \mathrm{~cm}$ from the bottom by a pencil. We have also drawn similar line across the plate below $1 \mathrm{~cm}$ from the upper edge of the plate. A spot were marked on the bottom line to drop the analytes. $50 \mu \mathrm{l}$ drops were put on the spot of the plate using thin capillary glass pipettes. Then we placed Plate in TLC tank (contained mobile phase; Acetone and Methanol: 1:1) and covered by lid and it was left until the mobile phase reached the upper line. UV detection box at $256 \mathrm{~nm}$ was used to visualize the spots. Spots marking were done by pencil for calculation of retention factor $(\mathrm{Rf})$.

\subsection{Calculation of $R \mathbf{R}$ values}

The $\mathrm{Rf}$ values are calculated with the distance travelled by the solvent, and the distance travelled by individual sample spots. Same Rf value of standard and sample considered similar compound. (Sarker et al., 2018). 


\subsection{Data analysis}

We have used Microsoft Excel-2010 to store data. Results were analyzed statistically using SPSS IMB 20 for descriptive statistics (IBM Corp. Released 2011, IBM SPSS Statistics for Windows, Version 20, Armonk, New York USA: IBM Corp) and graphs were prepared by Graph Pad Prism 6.

\section{Results}

\subsection{Questionnaire Survey to assess farmers' perspective}

Our survey covered different geographical location of Mymensingh division, mainly two districts: Mymensingh and Sheerpu. A total of thirty two farmers were surveyed from four upazillas (eight farms from each upazilla). We have selected the farms that were using colistin at the time of survey.

The constructed questionnaire was written both in English and Bangla. All surveyed farms were small scale broiler farms having flock size 500-2000 (Islam et al., 2010). Our data suggests most farmers are educated up to secondary level (46.87\%), $21.88 \%$ farmers had primary education, whereas $18.75 \%$ were higher secondary and $12.5 \%$ were Diploma/Graduate level education. Farmers confirmed that they use antibiotics both prophylactic and therapeutic purposes. Farmers keep the drugs in different places: Poultry shed (75\%), storage room (25\%). Only $9.37 \%$ farmers possess the knowledge of residual deposition. None of the farmers follow the withdrawal period. Every farmer collects their feed and chicks from commercial source, though farm management and hygiene were very poor in every farm visited.

\subsection{Percentage of colistin in different growing stages of broiler}

From each farm, nine samples were collected at the three different growth stages: 14-20 days (Early:E), 21-27 (Middle:M) days and 28-35 (Late:L) days, respectively. Out of 108 samples, 50\% (18 out of 36) samples were positive for TLC in early samples, whereas $66.67 \%$ (24 out of 36) samples for middle and 50\% (18 out of 36) for late collected samples (Figure 1).

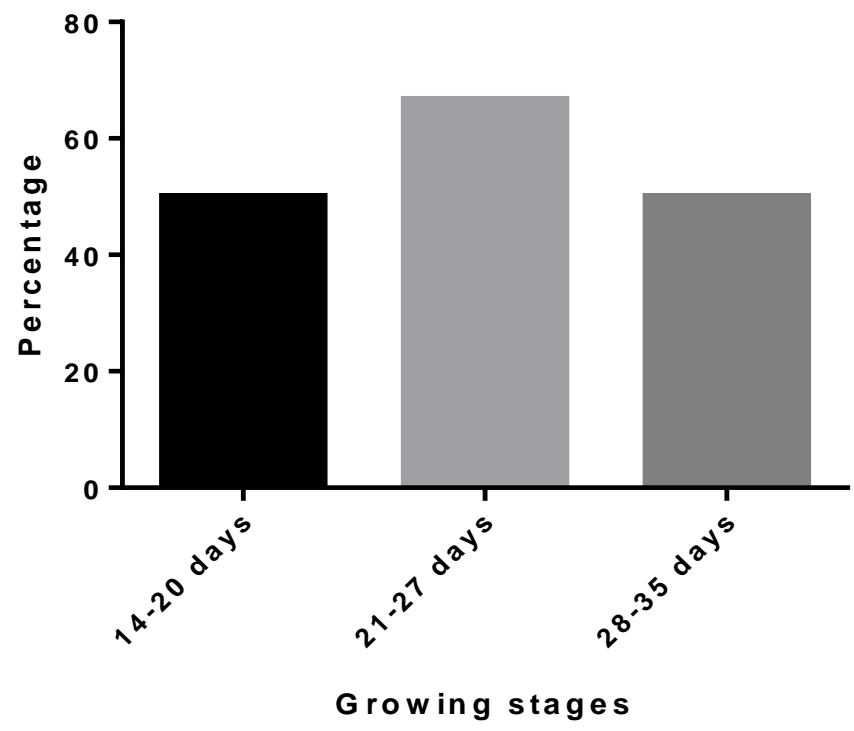

Figure 1. Presence of colistin residue in different growing stages.

\subsection{Presence of colistin in different geographical area}

A total of 108 samples, were collected from Fulbaria, Mymensingh Sadar, and Trishal Upazila of Mymensingh district and Nakla Upazila of Sherpur district. From each farm, nine samples were collected at the three different growth stages. The highest concentration was detected in Mymensingh sadar and Nakla (66.67\%) and lowest in Fulbaria (33.33\%). The results are shown in Figure 2. 


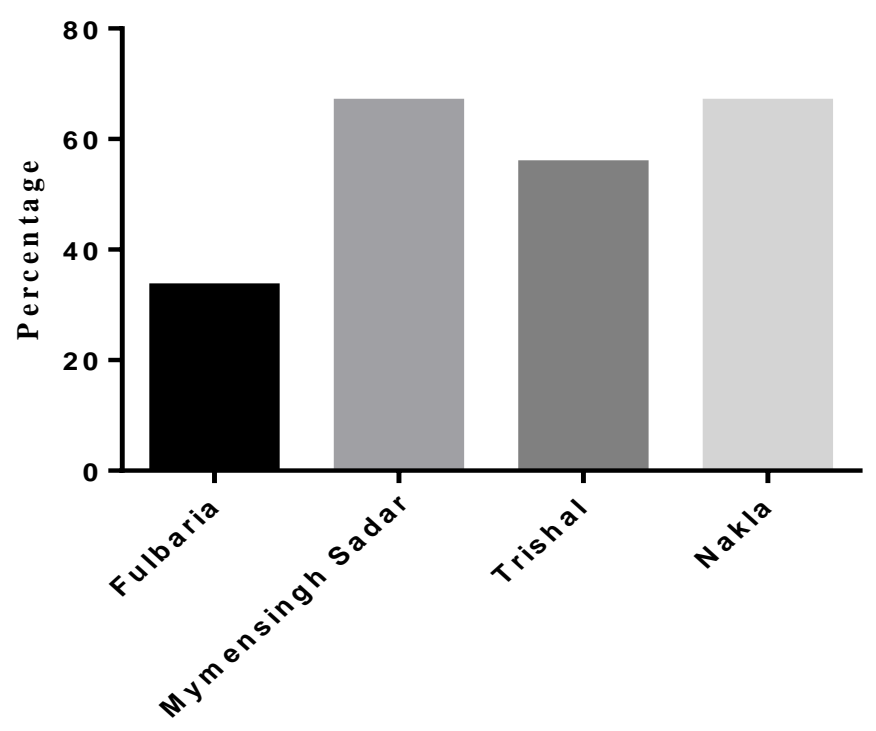

Figure 2. Presence of colistin residue in different farms at different geographical areas.

\section{Discussion}

In our study, questionnaire survey depicts that none of the respondent was practicing appropriate farm hygiene, biosecurity and withdrawal period of colistin. We haven't identified poor management with the farmers educational qualifications. Similar findings were described by Bashahun and Odoch, 2015; Krishnasamy et al., 2015 and Wadoum et al., 2016. We have observed that majority of the farmers continuously used antibiotics as therapeutic and prophylaxis and with greater intensity during disease outbreaks. These results do not completely differ from Nonga et al., 2009. However, disease surveillance data was not collected during the survey and no correlation was concluded.

We have detected colistin through TLC, one of the most used and effective technique. In our study we used TLC method to separate and identify colistin residue in broiler meat. In our study, 50\% (18 out of 36) samples were positive for TLC in early samples, whereas $66.67 \%$ (24 out of 36) samples for middle and 50\% (18 out of 36) for late collected samples (Figure 1). A similar type of study was conducted by Amjad et al., 2005 to separate and identify the ciprofloxacin and enrofloxacin residues from chicken liver, kidney and muscles using TLC.

The samples that were collected from Fulbaria, Mymensingh Sadar, Trishal and Nakla Upazila among them $33.33 \%, 66.67 \%, 55.56 \%$ and $66.67 \%$ samples were positive in TLC test (Figure 2). One or more than one antibiotics used in all selected farm. In Vietnam, a study conducted by Thuat et al., 2002 on antibiotic use in animal production and their residues in pork and chicken meat, showed that twenty six different antibiotics were used in pig and chicken production, from which, the seven more frequent were chloramphenicol in $15 \%$ of farm, tylosin (15\%), colistin (13\%), norfloxacin (10\%), gentamicin (8\%), tetracycline $(8 \%)$ and ampicillin (7\%).

Our study detected colistin in broiler in different growth stages and residue is continuing in food chain as withdrawal period is not being maintained. Strict measure should be taken to cease the residue in food chain to prevent drug resistance in human.

\section{Conclusions}

Antimicrobial agents are used for the prevention or treatment of animal diseases. In Bangladesh significant amount of antibiotics used in poultry farming but concerns have been raised that tissues of food animals contaminated with antimicrobial residues may cause adverse side effects in consumers. Evidence suggests that more judicious use of antibiotics in broiler production will reduce the residue level in the edible tissue and offal. Our study confirmed the presence of colistin residues in poultry meat samples collected from different Upazila of Mymensingh division. This may pose potential hazard to public health. Thus, it is recommended that measures should be taken to ensure observing proper withdrawal periods before marketing and drug control in veterinary use. 


\section{Acknowledgments}

The authors are highly obliged and expressing gratification and sincere appreciation the farm owners, who contributed towards the collection of data and samples.

\section{Conflict of interest}

None to declare.

\section{References}

Aamer M, AA Javaid and A Muhammad, 2000. Rational use of drugs in broiler meat production. Int. J. Agric. Biol., 2: 269-272.

Amjad H, I Javed and Muhammad N, 2005. Analysis of some residual antibiotics in muscle, kidney and liver samples of broiler chicken by various methods. Proceeding of the Pakistan Academy of Science. 42: 223231.

Bashahun D and TA Odoch, 2015. Assessment of antibiotic usage in intensive poultry farms in Wakiso District, Uganda. Livestock. Res. Rural. Dev., 27: 247.

Da Silva CA and M Rankin, 2014. Contract farming for inclusive market access. Food and Agriculture Organization of United Nation, Rome Italy., 227.

Hassan MM, KB Amin, M Ahaduzzaman, M Alam, MSA Faruk and I Uddin, 2014. Antimicrobial resistance pattern against E. coli and Salmonella in layer poultry. Research Journal for Veterinary Practitioners, 2: 3035 .

Higgins HC and JDG McEvoy, 2002. Accelerated solvent extraction of animal feeding stuffs for microbial growth inhibition screening for the presence of antimicrobial feed additives. Food. Addit. Contam., 19: 819828.

Hussain S, S Khan, J Ali, A Sultan, N Chand and Rafiullah, 2013. Antibiotic residues in commercial poultry meat and egg. International Workshop on Dairy Science Park, Pakistan. 2013.

Islam S, S Takashi and KQN Chhabi, 2010. Current scenario of the small-scale broiler farming in Bangladesh: potentials for the future projection. International Journal of Poultry Science, 9: 440-445.

Krishnasamy V, J Otte and E Silbergeld, 2015. Antimicrobial use in chinese swine and broiler poultry production. Antimicrobial Resistance and Infection Control, 4: 17.

Nisha A, 2008. Antibiotic residues-a global health hazard. Veterinary World, 1: 375.

Nonga H, M Mariki, ED Karimuribo and ED Mdegela, 2009. Assessment of antimicrobial usage and antimicrobial residues in broiler chickens in Morogoro Municipality, Tanzania. Pak. J. Nutr., 8: 203-207.

Okerman L, H Noppe, V Cornet and ZL De, 2007. Microbiological detection of 10 quinolone antibiotic residues and its application to artificially contaminated poultry samples. Food Additives and Contaminants, 24: 252257.

Popelka P, J Nagy, R Germuška, S Marcinčák, P Jevinova and RA De, 2005. Comparison of various assays used for detection of beta-lactam antibiotics in poultry meat. Food Additives and Contaminants, 22: 557-562.

Prabakaran R, 2003. Good practices in planning and management of integrated commercial poultry production in South Asia. FAO Animal Production and Health. Food and Agriculture Organization of the United Nation Rome, Italy

Sarker YA, MM Hasan, TK Paul, SZ Rashid, MN Alam and MH Sikder, 2018. Screening of antibiotic residues in chicken meat in Bangladesh by thin layer chromatography. Journal of Advanced Veterinary and Animal Research, 5: 140-145.

Singh S, S Shukla, N Tandia, N Kumar and R Paliwal, 2014. Antibiotic residues: a global challenge. Pharma Science Monitor, 5(3).

Tajick MA and B Shohreh, 2006. Detection of antibiotics residue in chicken meat using TLC. International Journal of Poultry Science, 5: 611-612.

Thuat DT, NN Tuan, VTT An, LT Hien, VB Lam and KT Ninh, 2002. Study on antibiotic use and antibiotic residues in pork and broiler meat of Binh Duong province. J. Vet. Sci., 50-57.

Wadoum REG, NF Zambou, FF Anyangwe, JR Njimou, MM Coman, MC Verdenelli, C Cecchini, S Silvi, C Orpianesi, A Cresci and V Colizzi, 2016. Abusive use of antibiotics in poultry farming in Cameroon and the public health implications. Br. Poult. Sci., $57: 483-493$.

Weiss C, A Conte, C Milandri, G Scortichini, P Semprini, R Usberti and G Migliorati, 2007. Veterinary drugs residue monitoring in Italian poultry : current strategies and possible developments. Food Control, 18: 10681076. 